\title{
Gentherapie
}

\section{Wie Designer-Rekombinasen Erbkrankheiten heilen könnten}

\author{
FELIX LANSING ${ }^{1}$, JENNA HOERSTEN ${ }^{1}$, FRANK BUCHHOLZ ${ }^{1,2}$ \\ ${ }^{1}$ MEDIZINISCHE SYSTEMBIOLOGIE (UCC-MSB), MEDIZINISCHE FAKULTÄT CARL \\ GUSTAV CARUS, TU DRESDEN \\ 2 NATIONALES CENTRUM FÜR TUMORERKRANKUNGEN (NCT/UCC), \\ PARTNERSTANDORT DRESDEN, TU DRESDEN
}

\section{Recent advances in nuclease-based genome editing allow for the cor- rection of many point-mutations causing diseases. However, correct- ing genetic alterations caused by larger chromosomal rearrangements remain challenging with this approach. Designer-recombinases prom- ise to fill this gap as demonstrated by the development of a hetero- dimeric Cre-based site-specific recombinase system. This system can functionally correct a large gene inversion frequently found in patients with severe Hemophilia A.}

DOI: $10.1007 / \mathrm{s} 12268-021-1549-9$

(C) Die Autoren 2021

Die Gentherapie erlebt derzeit einen bemerkenswerten Aufschwung. Hunderte neue wissenschaftliche Arbeiten und zahlreiche Firmengründungen belegen das akademische und kommerzielle Interesse an dieser Technologie. Dies liegt vor allem an der Entdeckung der Genschere CRISPR-Cas9 (clustered regularly interspaced short palindromic repeats und Cas9). Dieses System besteht aus zwei Komponenten, dem Cas9-Protein (eine Nuklease) und einer guide RNA (gRNA). Das Cas9-Protein kann mit einer geeigneten gRNA an fast jede Stelle des Genoms gelenkt werden und erzeugt dort einen gezielten DNA-Doppelstrangbruch [1]. Auch wenn es enorme Weiterentwicklungen der CRISPRSysteme gibt, ist ihre Anwendung, vor allem durch die Gefahr von ungewollten Genomveränderungen, für die Gentherapie immer noch risikobehaftet. Deshalb ist es von großer Bedeutung, auch andere molekulare Werkzeuge zur sicheren und präzisen Behandlung von Gendefekten zu entwickeln.

Designer-Rekombinasen als vielseitiges GenomeditierungsWerkzeug

Alternative Werkzeuge zur Genomeditierung sind sequenzspezifische Rekombinasen (SSRs) - eine Enzymklasse, die Genomverände- rungen durch DNA-Rekombination an bestimmten Erkennungssequenzen erlaubt [2]. SSRs haben einige Vorteile gegenüber dem CRISPR-Cas9-System. So bestehen sie aus nur einer Komponente, dem Rekombinase-Protein, und sind im Vergleich zum Cas9Protein ca. viermal kleiner. Dieser Größenunterschied erleichtert die Einschleusung in menschliche Zellen. Ein weiterer Vorteil, besonders bei therapeutischen Anwendungen, liegt darin, dass sie nicht auf zelleigene Reparaturmechanismen angewiesen sind. So führen sie DNA-Modifikationen mit äußerster Präzision und Effektivität durch. Außerdem sind SSRs sehr flexibel und können DNA-Fragmente sowohl ausschneiden als auch umdrehen oder austauschen. Damit besitzen sie ein breites Anwendungsspektrum (Abb. 1A).

Ein Nachteil von SSRs ist jedoch ihre zeitaufwendige Entwicklung für neue therapeutische Zielsequenzen. Anders als bei dem CRISPR-Cas9-System, das durch die gRNA zur Zielsequenz geleitet wird, ist das Rekombinase-Protein selbst für die DNA-Erkennung verantwortlich. Daher muss die Rekombinase für jede neue Anwendung verändert werden. Um dies zu erreichen, werden DesignerRekombinasen mithilfe der gerichteten Evolution für jede neue Zielsequenz „gezüchtet“
[3]. Ein erstes Beispiel für eine solche Designer-Rekombinase ist Brec1, die in einer klinischen Studie zur Behandlung von HIV genutzt werden soll [4].

Eine weitere Hürde für die Anwendung von SSRs in der Gentherapie ist die strikte Architektur der Zielsequenz. Diese besteht aus insgesamt 34 DNA-Basenpaaren (bp), wobei zwei 13 bp lange invers palindromische Sequenzen um eine zentrale 8-bpSpacersequenz angeordnet sind. Sie bilden eine spiegelverkehrte symmetrische Struktur. Wie man sich vorstellen kann, kommt eine Sequenz mit solch einer speziellen Architektur normalerweise nicht in unserem Genom vor.

Ein wichtiger Fortschritt bei der Generierung von SSRs wurde jüngst dadurch erreicht, dass die strikte symmetrische Architektur der Zielsequenz mit einem Trick umgangen werden konnte und damit die Anwendung von Designer-Rekombinasen vielfältiger geworden ist. Der Kunstgriff liegt darin, dass zwei verschiedene Rekombinasen (Heterodimer) anstelle von zwei gleichen Rekombinasen (Homodimer) eingesetzt werden. Dies ermöglicht die Adressierung von asymmetrischen, nicht invers palindromischen Zielsequenzen [5]. In diesem Szenario bindet jeweils eine Rekombinase die linke und die andere die rechte Zielsequenz. Gemeinsam lösen die beiden Rekombinasen dann die Rekombination aus (Abb. 1B).

\section{Korrektur einer Gen-Inversion bei Hämophilie A}

Als erste mögliche therapeutische Anwendung ist es uns unlängst gelungen, dieses Prinzip für eine asymmetrische Sequenz anzuwenden, die oft ursächlich für eine schwere Form der Hämophilie A verantwortlich ist. Hämophilie A ist die am häufigsten vorkommende Blutgerinnungsstörung und wird durch einen Defekt des Faktor-VIIIGens (F8) verursacht. Die momentane Behandlung basiert auf einer Proteinersatztherapie mit rekombinant hergestelltem Faktor VIII, der den betroffenen Patienten wöchentlich mehrmals intravenös verab- 
A

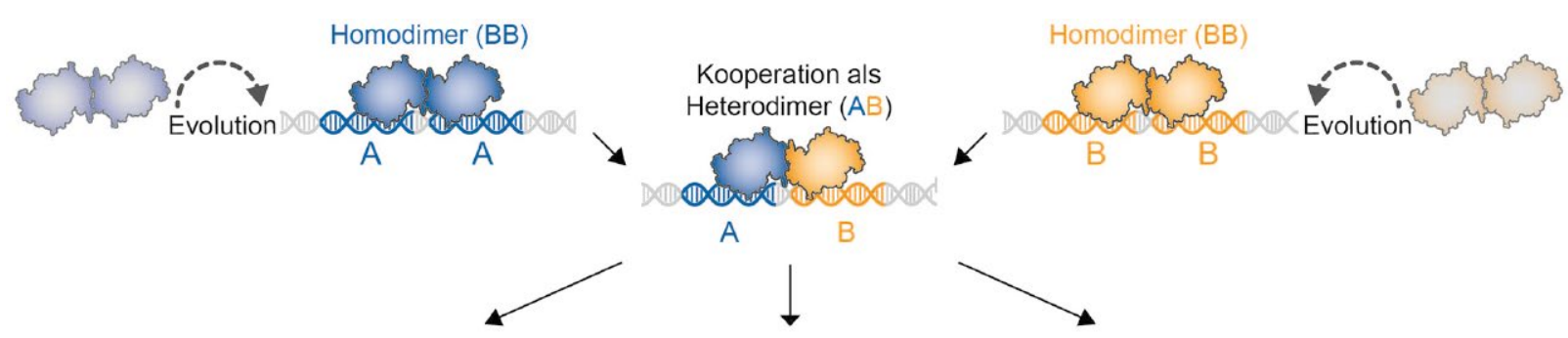

Deletion

Inversion

Austausch
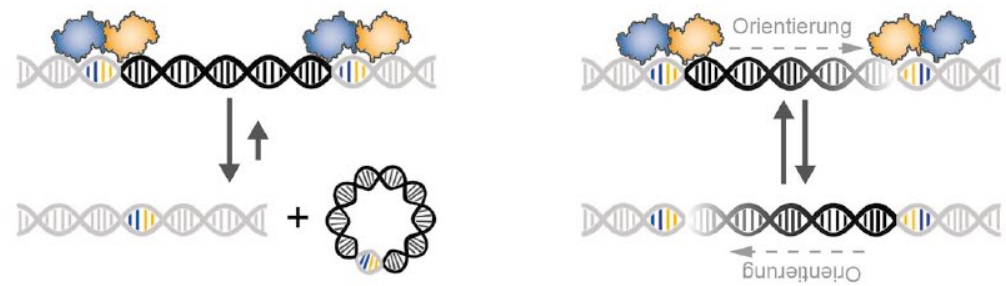

1

2

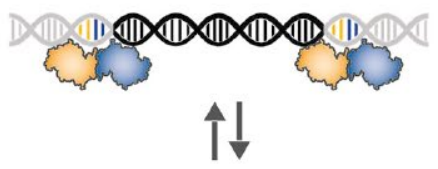

$A=$ Ziel-Sequenz $A$

$\mathrm{B}=$ Ziel-Sequenz $\mathrm{B}$

$\rightarrow=$ Reaktionsrichtung $1=$ Empfänger DNA
$=$ Rekombinase

2 = Austausch Fragment

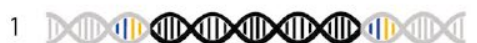

2 D(II)(II)(II)(II)(ID)(II)(II)(II)

B
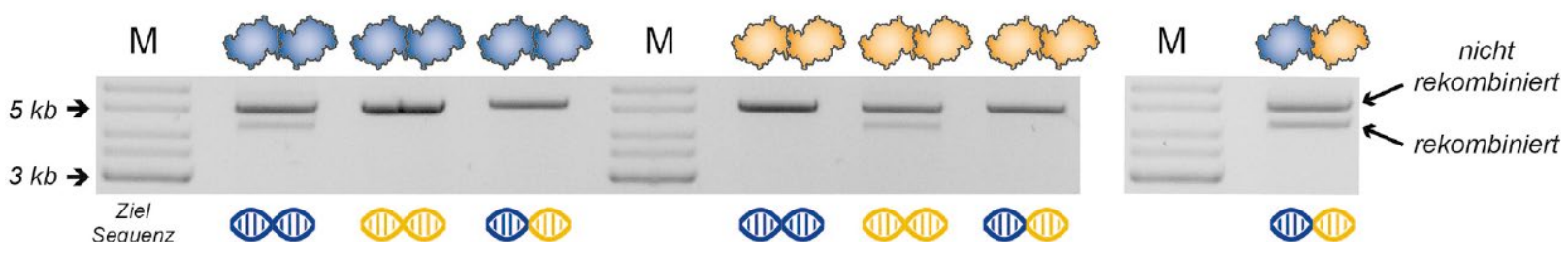

$\Delta$ Abb. 1: Entwicklung von heterospezifischen Designer-Rekombinasen. A, Darstellung der genetischen Veränderungen, die mit Designer-Rekombinasen vorgenommen werden können. Die gerichtete Evolution, um neue Designer-Rekombinasen herzustellen, ist durch einen unterbrochenen Pfeil dargestellt. Zwei unabhängig voneinander entwickelte Rekombinasen können zu Heterodimeren kombiniert werden. DNA-Fragmente können ausgeschnitten (Deletion), gedreht (Inversion) oder ausgetauscht (Austausch) werden. Die Farbcodierung (blau und orange) stellt die unterschiedlichen Rekombinasen und ihre jeweilige Zielsequenz dar. Die schwarz dargestellte DNA-Doppelhelix zwischen zwei Zielsequenzen wird dabei verändert. B, Experimenteller Rekombinationstest von Homo- und Heterodimeren. Die Aktivität (rekombiniert) von Homodimeren und Heterodimeren auf die jeweiligen Zielsequenzen wird durch eine kürzere DNA-Bande angezeigt. Die Homodimere rekombinieren spezifisch ihre symmetrische Zielsequenz (einfarbig). Nur in Kombination als Heterodimer wird die asymmetrische Zielsequenz rekombiniert (zweifarbig). M: Marker; Kb: Kilobasen-Paare.

reicht werden muss. Durch die notwendige regelmäßige Verabreichung des Faktor-VIIIProteins büßen Patienten unersetzbare Lebensqualität ein. Weiterhin wird das Gesundheitssystem durch die hohen Kosten von 100.000-900.000 Euro pro Jahr und Patient belastet [6]

Bei etwa der Hälfte der schwer erkrankten Hämophilie-A-Patienten liegt eine Mutation zugrunde, die das F8-Gen strukturell umstellt, eine Gen-Inversion [7]. Um diesen Patienten zu helfen, wäre daher ein genetisches Werkzeug nötig, das Gen-Inversionen korrigieren kann. Dies kann prinzipiell gelingen, wenn mit Nukleasen zwei DNA-Doppelstrangbrüche in geeigneten Regionen des Genoms herbeigeführt werden. Wenn bei der Reparatur die Zelle dann zufällig das DNAFragment dreht, kann es zur Korrektur der Gen-Inversion kommen. Wie man sich leicht vorstellen kann, ist dieser Prozess aber ineffektiv. Deshalb ist es schwierig, GenInversionen mithilfe von Nukleasen zu korrigieren.

Rekombinasen hingegen sind bekannt dafür, dass sie auch große DNA-Fragmente effizient und präzise invertieren können [8]. Es gibt jedoch keine natürlich vorkommende Rekombinase, die zur Behandlung von Hämophilie-A-Patienten genutzt werden könnte. Daher haben wir nach Analyse der genomischen Sequenz des F8-Gens ein heterodimeres SSR-System entwickelt, das auf den im menschlichen Genom natürlich vorkommenden Sequenzen agiert (Abb. 2). Tatsächlich konnten wir nachweisen, dass diese Designer-Rekombinase (RecF8) in menschlichen Zellen aktiv ist und die Zielsequenz spezifisch und effizient rekombiniert. Weitere Tests zeigten, dass RecF8 in der Lage ist, die Gen-Inversion in Zellen von einem Hämophilie-A-Patienten rückgängig zu machen.
Die genetische Korrektur führte in den Patientenzellen dabei tatsächlich auch zur Expression von Faktor-VIII-Boten-RNA. Diese Ergebnisse zeigen zum ersten Mal, dass es möglich ist, Designer-Rekombinasen zur Korrektur von krankheitsauslösenden GenInversionen zu nutzten [9].

\section{Ausblick}

Das Potenzial der Genomeditierung ist immens und bietet erstmalig eine Heilungschance für ansonsten als unheilbar geltende genetische Erkrankungen. Die ursächliche genetische Vielfalt der Erkrankungen spiegelt den hohen Bedarf an maßgeschneiderten Therapien wider. Jede einzelne Erkrankung muss mit dem bestmöglichen Gen-Werkzeug behandelt werden. Hierbei können DesignerRekombinasen eine wichtige Rolle einnehmen. So sind Rekombinasen besonders gut geeignet, z. B. für die Korrektur von Gen- 


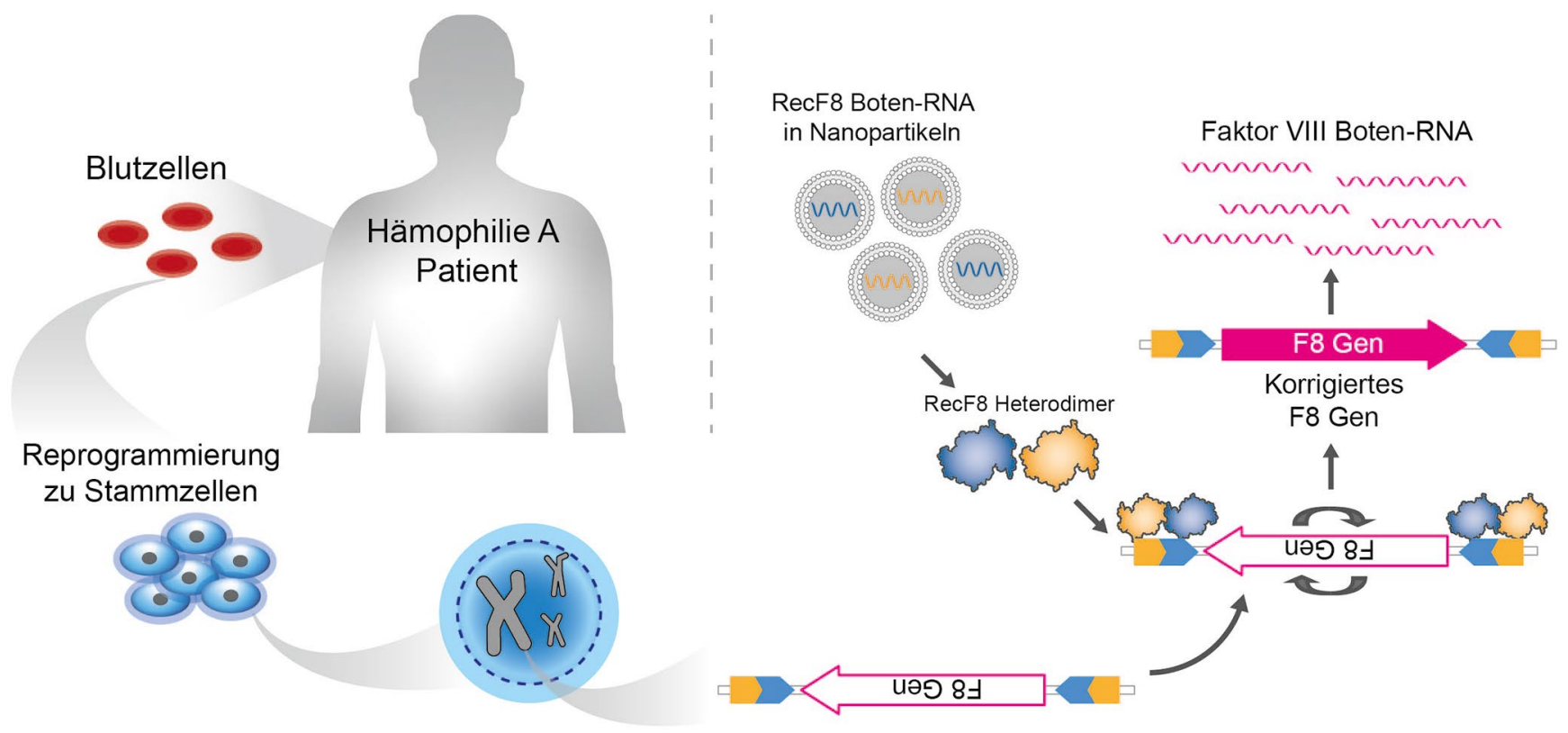

$\Delta$ Abb. 2: Schematische Darstellung zur Korrektur der Gen-Inversion im F8-Gen mit der heterodimeren Designer-Rekombinase RecF8. Der Ablauf der einzelnen Schritte ist durch Pfeile dargestellt.

Inversionen - wie am Beispiel der Hämophilie A gezeigt. Bevor jedoch eine klinische Testung mit menschlichen Probanden begonnen werden kann, muss zunächst die Sicherheit von RecF8 im Tiermodell bestätigt werden. Sollte sich die Spezifizität und Effizienz, die in humanen Zellen zu beobachten war, auch im Tiermodell zeigen, könnten vielleicht schon bald Hämophilie-A-Patienten ihr eigenes Faktor-VIII-Protein im Körper produzieren.

\section{Danksagung}

Wir danken allen Mitgliedern des Buchholz Labors für die Unterstützung dieser Arbeit. Ein besonderer Dank gilt dem Spender und dessen Familie für die Bereitstellung der Blutprobe.

\section{Literatur}

[1] Komor AC, Badran AH, Liu DR (2017) CRISPR-based technologies for the manipulation of eukaryotic genomes. Cell 168: 20-36

[2] Meinke G, Bohm A, Hauber J et al. (2016) Cre recombinase and other tyrosine recombinases. Chem Rev 116: 12785-12820

[3] Buchholz F, Stewart AF (2001) Alteration of Cre recombinase site specificity by substrate-linked protein evolution.

Nat Biotechnol 19: 1047-1052

[4] Karpinski J, Hauber I, Chemnitz J et al. (2016), Directed evolution of a recombinase that excises the provirus of most HIV-1 primary isolates with high specificity. Nat Biotechnol 34: 401-409

[5] Lansing F, Paszkowski-Rogacz M, Schmitt LT et al. (2020) A heterodimer of evolved designer-recombinases precisely excises a human genomic DNA locus. Nucleic Acids Res 48: 472-485

[6] Croteau SE, Cheng D, Cohen AJ et al. (2019) Regional variation and cost implications of prescribed extended halflife factor concentrates among U.S. Haemophilia Treatment Centres for patients with moderate and severe haemophilia. Haemophilia 25: 668-675
[7] Lakich D, Kazazian HH, Antonarakis SE et al. (1993) cause of severe haemophilia A. Nat Genet 5: 236-241 [8] Zheng B, Sage M, Sheppeard EA et al. (2000) Engineering mouse chromosomes with Cre-loxP: range, efficiency, and somatic applications. Mol Cell Biol 20: 648-655 [9] Lansing F, Mukhametzyanova L, Rojo Romanos T et al. (2020) Correction of a factor VIII genomic inversion with designer-recombinases. bioRxiv, doi: https://doi.org/ 10.1101/2020.11.02.328013

Funding note: Open Access funding enabled and organized by Projekt DEAL. Open Access: Dieser Artikel wird unter der Creative Commons Namensnennu

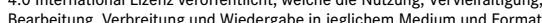
Bearbeitung, Verbreitung und Wiedergabe in jeglichem Medium und ordnungsgemäß nennen, einen Link zur Creative Commons Lizenz beifügen und angeben, ob Änderungen vorgenommen wurden. Die in diesem Artikel enthaltenen Bilder und sonstiges Drittmaterial unterliegen ebenfalls der Inversions disrupting the factor VIII gene are a common

genannten Creative Commons Lizenz, sofern sich aus der Abbildungslegende nichts anderes ergibt. Sofern das betreffende Material nicht unter der genannten Creative Commons Lizenz stent und die betreffende Handlung nich Weiterverwendungen des Materials die Einwilligung des jeweiligen Rechteinhabers einzuholen. Weitere Details zur Lizenz entnehmen Sie bitte der Lizenzinformation auf http://creativecommons.org/licenses/by/4.0/deed.de.

\section{Korrespondenzadresse:}

Prof. Dr. Frank Buchholz

Medizinische Systembiologie (UCC-MSB)

Medizinische Fakultät Carl Gustav Carus

Technische Universität Dresden

Fetscherstraße 74

D-01307 Dresden

frank.buchholz@tu-dresden.de

\section{AUTOREN}

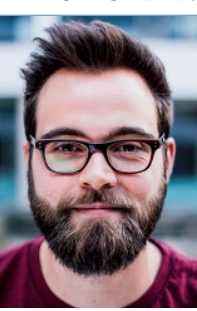

Felix Lansing

Jahrgang 1990. 2011-2016 B. Sc. Molekularbiologie, Universität Bielefeld, und

M. Sc. Regenerative Biology and Medicine, CRTD, TU Dresden. Seit 2017 Promotion

über die Entwicklung von Designer-Rekombinasen zur Genomchirurgie.

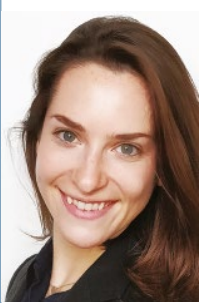

Jenna Hoersten

Jahrgang 1990. 2008-2013 B. Sc. Biomedical Engineering, Ohio State University,

USA. 2013-2015 Datenmanagment bei JP Morgan \& Chase, Columbus, OH, USA.

2016-2018 M. Sc. Molecular Bioengineering, TU Dresden. Seit 2019 erst Forschungsassistenz und später Promotion zur Spezifitätsverbesserung und Off-Target-Vorhersagen von Designer-Rekombinasen.

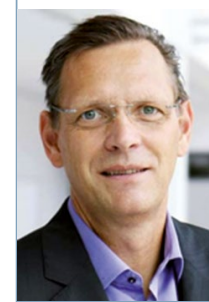

Frank Buchholz

Jahrgang 1966. Biologiestudium an der Universität Göttingen. 1998 Promotion am EMBL Heidelberg. 1998-2002 Postdoc an der University of California, San Francisco, USA. 2002-2010 Gruppenleiter am Max-Plank-Instut für molekulare Zellbiologie und Genetik, Dresden. Seit 2010 Professor und Arbeitsgruppenleiter der Medizinischen Systembiologie und Leiter der translationalen Forschung am NCT/UCC an der Medizinischen Fakultät der TU Dresden. 